\title{
MEASURES OF DEPENDENCE FOR STABLE AR(1) MODELS WITH TIME-VARYING COEFFICIENTS
}

\section{J. Nowicka-Zagrajek and A. Wyłomańska}

Institute of Mathematics and Computer Science, Wroctaw University of Technology, Poland

\begin{abstract}
$\square \quad$ In this paper we study the dependence structure for $A R(1)$ models with symmetric $\alpha$-stable innovations and time-varying coefficients. In this case the covariance function is not defined and therefore other measures of dependence have to be used. We formulate conditions providing the bounded solutions of such systems and give the form of the solution. Then the covariation and the codifference for stable $A R(1)$ models are studied and the asymptotic relation between these two measures of dependence for the considered models is examined.
\end{abstract}

Keywords AR models; Codifference; Covariation; Dependence structure; Stable random variables.

AMS Subject Classification 60G52; 60H35; $91 \mathrm{~B} 70$.

\section{INTRODUCTION}

Many studies have shown that heavy-tailed distributions allow for modelling different kinds of phenomena when the assumption of normality for the observations seems not to be reasonable. Especially $\alpha$-stable (stable) distributions have found many practical applications, for instance in finance (Mittnik and Rachev ${ }^{[6]}$ ), physics (Janicki and Weron $^{[3]}$ ), and electrical engineering (Stuck and Kleiner ${ }^{[13]}$ ). The importance of this class of distributions is strongly supported by the limit theorems that indicate that the stable distribution is the only possible limiting distribution for the normed sum of independent and identically distributed random variables.

PARMA (Periodic Autoregressive Moving Average) models with Gaussian innovations-a special case of ARMA models with time-varying

Received November 2006; Accepted November 2007

Address correspondence to J. Nowicka-Zagrajek, Institute of Mathematics and Computer Science, Wrocław University of Technology, Wrocław, Poland; E-mail: joanna.nowickazagrajek@pwr.wroc.pl 
coefficients - were introduced as a class of periodically correlated processes (see Gladyshev ${ }^{[2]}$ ). These models have received much attention in the literature as they can be applied to modelling many phenomena in various areas, e.g., in hydrology (Vecchia ${ }^{[14,15]}$ ), meteorology (Jones and Brelsford $^{[4]}$, Monin ${ }^{[7]}$ ), economics (Parzen and Pagano ${ }^{[11]}$ ) and electrical engineering (Gardner and Franks ${ }^{[1]}$ ).

ARMA models with varying coefficients and symmetric stable innovations combine the advantages of ARMA models with timedependent coefficients and stable distributions-they offer an alternative for modelling non-stationary time series with heavy tails. However, in this case the covariance function is not defined and thus other measures of dependence have to be used. The most popular measures-the covariation and the codifference-were examined in Nowicka ${ }^{[8]}$ and Nowicka and Weron $^{[9]}$ for stationary ARMA models with stable innovations and with constant coefficients. The results concerning the asymptotic relation between the covariation and the codifference obtained in these papers led to subsequent studies. The behaviour of the measures of dependence for PAR(1) models (i.e., AR with periodic coefficients) was investigated in Nowicka-Zagrajek and Wyłomańska ${ }^{[10]}$.

In this paper we study the $\operatorname{AR}(1)$ systems with $\alpha$-stable innovations, i.e., time series satisfying the following equations:

$$
X_{n}-b_{n} X_{n-1}=a_{n} \xi_{n}, \quad n \in Z
$$

where, in contrast to Nowicka ${ }^{[8]}$ and Nowicka and Weron ${ }^{[9]}$, the nonzero real coefficients $\left\{b_{n}\right\}$ and $\left\{a_{n}\right\}$ are not constant and the innovations $\left\{\xi_{n}\right\}$ are independent symmetric $\alpha$-stable ( $\mathrm{S} \alpha \mathrm{S}$ for short) random variables given by the following characteristic function:

$$
\mathbb{E} \exp \left\{i t \xi_{n}\right\}=\exp \left\{-\sigma_{\xi}^{\alpha}|t|^{\alpha}\right\}, \quad 0<\alpha \leq 2,
$$

where $\sigma_{\xi}$ denotes the scale parameter.

Moreover, we assume that system (1.1) satisfies one of the following conditions:

$$
\begin{gathered}
\lim _{r \rightarrow-\infty}\left|B_{r}^{0}\right|=0 \quad \text { and } \quad \sup _{n \in Z} \sum_{s=0}^{\infty}\left|B_{n-s+1}^{n} a_{n-s}\right|^{\alpha}<\infty \\
\lim _{r \rightarrow \infty}\left|B_{0}^{r}\right|^{-1}=0 \text { and } \sup _{n \in Z} \sum_{s=1}^{\infty}\left|\frac{a_{n+s}}{B_{n+1}^{n+s}}\right|^{\alpha}<\infty
\end{gathered}
$$

where

$$
B_{r}^{s}=\prod_{j=r}^{s} b_{j}
$$


with the convention $B_{r}^{s}=1$ if $r>s$. Conditions (I) and (II) provide the bounded solution of the system (1.1). As the covariance function is not defined for stable random vectors, in Section 2 we present two other measures of dependence that can be used for symmetric stable time series-the covariation and the codifference. The covariation and the codifference for $\mathrm{AR}(1)$ models with stable innovations are studied in Sections 3 and 4 for conditions (I) and (II), respectively, and the asymptotic relation between these two measures of dependence for the considered models is examined there. We point out that conditions (I) and (II) lead to the bounded solution of $\mathrm{AR}(1)$ models with $\mathrm{S} \alpha \mathrm{S}$ innovations and we provide the form of this solution. In order to illustrate theoretical results, in Sections 3 and 4 we give examples of $\mathrm{AR}(1)$ systems with $\mathrm{S} \alpha \mathrm{S}$ innovations. These examples also show the difference between the asymptotic behaviour of the considered measures of dependence in the case of constant coefficients and time-varying coefficients.

\section{MEASURES OF DEPENDENCE FOR STABLE TIME SERIES}

Let $X$ and $Y$ be jointly $\mathrm{S} \alpha \mathrm{S}$ and let $\Gamma$ be the spectral measure of the random vector $(X, Y)$ (see, for instance, Samorodnitsky and $\left.\mathrm{Taqqu}^{[12]}\right)$. If $\alpha<2$ then the covariance is not defined and thus other measures of dependence have to be used. The most popular measures are the covariation $C V(X, Y)$ and the codifference $C D(X, Y)$ given in Definitions 2.1 and 2.2, respectively.

Definition 2.1. Let $X$ and $Y$ be jointly $\mathrm{S} \alpha \mathrm{S}$. The covariation $C V(X, Y)$ of $X$ on $Y$ defined for $1<\alpha \leq 2$ is the real number

$$
C V(X, Y)=\int_{S_{2}} s_{1} s_{2}^{\langle\alpha-1\rangle} \Gamma(d \mathbf{s}),
$$

where $\Gamma$ is the spectral measure of the random vector $(X, Y), S_{2}$ is the unit sphere in $\mathbb{R}^{2}, \mathbf{s}=\left(s_{1}, s_{2}\right)$ and the signed power $z^{\langle p\rangle}$ is given by $z^{\langle p\rangle}=$ $|z|^{p} \operatorname{sign}(z)$.

Definition 2.2. Let $X$ and $Y$ be jointly $\mathrm{S} \alpha \mathrm{S}$. The codifference $C D(X, Y)$ of $X$ on $Y$ defined for $0<\alpha \leq 2$ equals

$$
C D(X, Y)=\ln \mathbb{E} \exp \{i(X-Y)\}-\ln \mathbb{E} \exp \{i X\}-\ln \mathbb{E} \exp \{-i Y\} .
$$

Properties of the considered measures of dependence one can find in Samorodnitsky and Taqqu ${ }^{[12]}$. Let us only mention here that, in contrast 
to the codifference, the covariation is not symmetric in its arguments. Moreover, when $\alpha=2$ both measures reduce to the covariance, namely

$$
\operatorname{Cov}(X, Y)=2 C V(X, Y)=C D(X, Y)
$$

If $\alpha>1$, then the covariation induces a norm $\|\cdot\|_{\alpha}$ on the linear space $\mathrm{S}_{\alpha}$ of jointly $\mathrm{S} \alpha \mathrm{S}$ random variables.

Definition 2.3. The covariation norm of $X \in \mathrm{S}_{\alpha}, \alpha>1$, is

$$
\|X\|_{\alpha}=(C V(X, X))^{1 / \alpha}
$$

The covariation norm (norm for short) of a $\mathrm{S} \alpha \mathrm{S}$ random variable $X$ is equal to the scale parameter of this variable, i.e., $\|X\|_{\alpha}=\sigma_{X}$; see Samorodnitsky and Taqqu ${ }^{[12]}$. Let us recall that $X=Y$ in $S_{\alpha}$ if and only if $\|X-Y\|_{\alpha}=0$ and that the sequence $\left\{X_{n}\right\}_{n \in Z}$ is bounded in a space $S_{\alpha}$ if $\sup _{n \in Z}\left\|X_{n}\right\|_{\alpha}^{\alpha}<\infty$.

As the covariation and the covariation norm are defined for $\alpha>1$ we will make the assumption that $1<\alpha \leq 2$ throughout this paper. Then the codifference can be rewritten in the form

$$
C D(X, Y)=\|X\|_{\alpha}^{\alpha}+\|Y\|_{\alpha}^{\alpha}-\|X-Y\|_{\alpha}^{\alpha}
$$

\section{AR(1) MODELS SATISFYING CONDITION (I)}

Let us assume that condition (I) is satisfied and $1<\alpha \leq 2$. In this case it is not difficult to show that $\operatorname{AR}(1)$ system defined by (1.1) with $\mathrm{S} \alpha \mathrm{S}$ innovations has the bounded solution in the space $S_{\alpha}$ with norm $\|.\|_{\alpha}$ given by the formula:

$$
X_{n}=\sum_{s=0}^{\infty} B_{n-s+1}^{n} a_{n-s} \xi_{n-s}
$$

and $X_{n}$ converges a.s. This means that results obtained in Makagon et al. ${ }^{[5]}$ for ARMA systems with Gaussian innovations can be extended to the case of stable innovations.

The moving average representation (3.3) is very convenient because both the covariation and the codifference can be expressed in terms of the coefficients.

Proposition 3.1. If $\quad \lim _{r \rightarrow-\infty}\left|B_{r}^{0}\right|=0, \quad \sup _{n \in Z} \sum_{s=0}^{\infty}\left|B_{n-s+1}^{n} a_{n-s}\right|^{\alpha}<\infty$ (condition (I)) and $\left\{X_{n}\right\}$ is the bounded solution of (1.1), then for $1<\alpha \leq 2$ : 
(i) the covariation of $X_{n}$ on $X_{m}(n, m \in Z)$ has the following form:

$$
C V\left(X_{n}, X_{m}\right)=\sigma_{\xi}^{\alpha}\left(B_{\min (n, m)+1}^{\max (n, m)}\right)^{\operatorname{sign}(n-m)} \sum_{s=\max (0, n-m)}^{\infty}\left|B_{n-s+1}^{m} a_{n-s}\right|^{\alpha},
$$

(ii) the codifference of $X_{n}$ on $X_{m}(n, m \in Z)$ has the following form:

$$
\begin{aligned}
C D\left(X_{n}, X_{m}\right)= & \sigma_{\xi}^{\alpha}\left(1+\left|B_{\min (n, m)+1}^{\max (n, m)}\right|^{\alpha}-\left|1-\left(B_{\min (n, m)+1}^{\max (n, m)}\right)\right|^{\alpha}\right) \\
& \times \sum_{s=|m-n|}^{\infty}\left|B_{\max (n, m)-s+1}^{\min (n, m)} a_{\max (n, m)-s}\right|^{\alpha} \\
= & \sigma_{\xi}^{\alpha}\left(1+\left|B_{\min (n, m)+1}^{\max (n, m)}\right|^{\alpha \cdot \operatorname{sign}(n-m)}-\left|1-\left(B_{\min (n, m)+1}^{\max (n, m)}\right)^{\operatorname{sign}(n-m)}\right|^{\alpha}\right) \\
& \times \sum_{s=\max (0, n-m)}^{\infty}\left|B_{n-s+1}^{m} a_{n-s}\right|^{\alpha} .
\end{aligned}
$$

Proof. Formulae (3.4) and (3.5) are the consequences of Proposition 3.5.2 in Samorodnitsky and Taqqu ${ }^{[12]}$.

Example 3.1 (The Case of Constant Coefficients). Let us consider the special case of $\mathrm{AR}(1)$ system, i.e., model with the constant coefficients, given by the equation:

$$
X_{n}-b X_{n-1}=a \xi_{n}
$$

where $a, b \neq 0,|b|<1$ are real numbers and $\left\{\xi_{n}\right\}$ are independent $\mathrm{S} \alpha \mathrm{S}$ innovations with the scale parameter $\sigma_{\xi}$. Note, that if $a=1$, then we have $\mathrm{AR}(1)$ model considered in Nowicka and Weron ${ }^{[9]}$.

Now $a_{n-s}=a$ and $B_{n-s+1}^{n}=b^{s}$ so it is easy to show that $\lim _{r \rightarrow-\infty}\left|B_{r}^{0}\right|^{-1}=$ $\lim _{r \rightarrow-\infty}|b|^{-r+1}=0, \sup _{n \in Z} \sum_{s=0}^{\infty}\left|B_{n-s+1}^{n} a_{n-s}\right|^{\alpha}=|a|^{\alpha} /\left(1-|b|^{\alpha}\right)<\infty$. Therefore, condition (I) is satisfied and

$$
X_{n}=a \sum_{s=0}^{\infty} b^{s} \xi_{n-s}
$$

Applying Proposition 3.1 we obtain for $1<\alpha \leq 2$ :

$$
\begin{aligned}
& C V\left(X_{n}, X_{n-k}\right)=C V\left(X_{n+k}, X_{n}\right)=\frac{\sigma_{\xi}^{\alpha} b^{k}|a|^{\alpha}}{1-|b|^{\alpha}}, \\
& C V\left(X_{n}, X_{n+k}\right)=C V\left(X_{n-k}, X_{n}\right)=\frac{\sigma_{\xi}^{\alpha}|b|^{\alpha k}|a|^{\alpha}}{b^{k}\left(1-|b|^{\alpha}\right)},
\end{aligned}
$$




$$
\begin{aligned}
C D\left(X_{n}, X_{n-k}\right) & =C D\left(X_{n+k}, X_{n}\right)=C D\left(X_{n}, X_{n+k}\right)=C D\left(X_{n-k}, X_{n}\right) \\
& =\frac{\sigma_{\xi}^{\alpha}\left(1+|b|^{\alpha k}-\left|1-b^{k}\right|^{\alpha}\right)|a|^{\alpha}}{1-|b|^{\alpha}},
\end{aligned}
$$

for every $n \in Z$ and for $k \in Z, k>0$. Results (3.6)-(3.8) are not surprising because of the stationarity of $X_{n}$.

Now let us recall the fact that for $|b|<1$ and $1<\alpha<2$ we have: $\frac{1-\left|1-b^{k}\right|^{\alpha}}{b^{k}} \rightarrow \alpha, \frac{|b|^{k \alpha}}{b^{k}} \rightarrow 0$, and $\frac{b^{2 k}}{|b|^{k \alpha}} \rightarrow 0$ as $k \rightarrow \infty$. Thus it is not difficult to show that for $1<\alpha<2$ and for every $n$ we obtain the following limits:
(a) $\lim _{k \rightarrow \infty} \frac{C D\left(X_{n+k}, X_{n}\right)}{C V\left(X_{n+k}, X_{n}\right)}=\alpha$,
(c) $\lim _{k \rightarrow \infty} \frac{C D\left(X_{n-k}, X_{n}\right)}{C V\left(X_{n-k}, X_{n}\right)}=0$,
(b) $\lim _{k \rightarrow \infty} \frac{C D\left(X_{n}, X_{n-k}\right)}{C V\left(X_{n}, X_{n-k}\right)}=\alpha$,
(d) $\lim _{k \rightarrow \infty} \frac{C D\left(X_{n}, X_{n+k}\right)}{C V\left(X_{n}, X_{n+k}\right)}=0$.

If $a=1$, then the first result is the same as in Theorem 3.3 in Nowicka and Weron ${ }^{[9]}$. Moreover, cases (a) and (b) show that Theorem 3.3 in Nowicka and Weron ${ }^{[9]}$ can be extended; see Corollary 3.1. The other results give us the argument, that $C D$ is not always asymptotically proportional to $\alpha C V$. It is worth pointing out here that the results $(\mathrm{a})-(\mathrm{d})$ are true for PAR(1) models (i.e., AR models with periodic coefficients) with stable innovations; see Nowicka-Zagrajek and Wyłomańska ${ }^{[10]}$.

Corollary 3.1. For the stationary time series given by the equation $X_{n}-b X_{n-1}=$ $\xi_{n}$, where $|b|<1$ is a real number and $\left\{\xi_{n}\right\}$ are independent $\mathrm{S} \alpha \mathrm{S}$ innovations with scale parameter $\sigma_{\xi}$ and $1<\alpha \leq 2$ the following formula holds:

$$
\lim _{k \rightarrow \infty} \frac{C D\left(X_{k}, X_{0}\right)}{C V\left(X_{k}, X_{0}\right)}=\lim _{k \rightarrow \infty} \frac{C D\left(X_{0}, X_{-k}\right)}{C V\left(X_{0}, X_{-k}\right)}=\alpha .
$$

Now the following question arises: Are the results $(a)-(d)$ in Example 3.1 true in general in the case of $\mathrm{AR}(1)$ models satisfying condition (I)? The answer is stated in Theorem 3.1 and in Example 3.2.

Theorem 3.1. If $\lim _{r \rightarrow-\infty}\left|B_{r}^{0}\right|=0, \sup _{n \in Z} \sum_{s=0}^{\infty}\left|B_{n-s+1}^{n} a_{n-s}\right|^{\alpha}<\infty \quad$ (condition (I)) and $\left\{X_{n}\right\}$ is the bounded solution of system (1.1), then:

(a) for $1<\alpha \leq 2$ and $n \in Z$ the following formula holds:

$$
\lim _{k \rightarrow \infty} \frac{C D\left(X_{n}, X_{n-k}\right)}{C V\left(X_{n}, X_{n-k}\right)}=\alpha,
$$


(b) for $1<\alpha<2$ and $n \in Z$ the following formula holds:

$$
\lim _{k \rightarrow \infty} \frac{C D\left(X_{n-k}, X_{n}\right)}{C V\left(X_{n-k}, X_{n}\right)}=0
$$

Proof. First, let us note that by assumption $\forall n \in Z, b_{n} \neq 0$ and condition (I), $\lim _{k \rightarrow \infty}\left|B_{n-k+1}^{n}\right|=0$ holds for each $n \in Z$. obtain:

(a) Applying Proposition 3.1 for $1<\alpha \leq 2, n, k \in Z$, and $k>0$ we

$$
\frac{C D\left(X_{n}, X_{n-k}\right)}{C V\left(X_{n}, X_{n-k}\right)}=\frac{1+\left|B_{n-k+1}^{n}\right|^{\alpha}-\left|1-B_{n-k+1}^{n}\right|^{\alpha}}{B_{n-k+1}^{n}} .
$$

Since for $1<\alpha \leq 2$ we have:

$$
\lim _{x \rightarrow 0} \frac{1-|1-x|^{\alpha}}{x}=\alpha, \quad \lim _{x \rightarrow 0} \frac{|x|^{\alpha}}{x}=0,
$$

for $1<\alpha \leq 2$ and $n \in Z$ we see that:

$$
\lim _{k \rightarrow \infty} \frac{C D\left(X_{n}, X_{n-k}\right)}{C V\left(X_{n}, X_{n-k}\right)}=\lim _{k \rightarrow \infty} \frac{1-\left|1-B_{n-k+1}^{n}\right|^{\alpha}}{B_{n-k+1}^{n}}+\lim _{k \rightarrow \infty} \frac{\left|B_{n-k+1}^{n}\right|^{\alpha}}{B_{n-k+1}^{n}}=\alpha .
$$

(b) By Proposition 3.1 for $1<\alpha \leq 2, n, k \in Z$, and $k>0$ we get:

$$
\frac{C D\left(X_{n-k}, X_{n}\right)}{C V\left(X_{n-k}, X_{n}\right)}=\frac{1+\frac{1}{\left|B_{n-k+1}^{n}\right|^{\alpha}}-\left|1-\frac{1}{B_{n-k+1}^{n}}\right|^{\alpha}}{\frac{1}{B_{n-k+1}^{n}}}
$$

To complete the proof it is sufficient to note that for $1<\alpha<2$

$$
\lim _{x \rightarrow 0} \frac{1+\frac{1}{|x|^{\alpha}}-\left|1-\frac{1}{x}\right|^{\alpha}}{\frac{1}{x}}=0
$$

The other two cases are not true in general-it is enough to analyze Example 3.2 to see that we get the results that differ from those obtained in Example 3.1.

Example 3.2. Let us take into account the $\mathrm{AR}(1)$ system given by (1.1) with $\mathrm{S} \alpha \mathrm{S}$ innovations given by (1.2) and discuss two examples of coefficients.

(a) The coefficients are given by

$$
b_{n}=\left\{\begin{array}{ll}
1 & \text { for } n \geq 0, \\
\frac{1}{\sqrt[4]{2}} & \text { for } n<0,
\end{array} \quad a_{n}= \begin{cases}\left(\frac{1}{2}\right)^{n / \alpha} & \text { for } n \geq 0 \\
1 & \text { for } n<0\end{cases}\right.
$$


It follows easily that $\lim _{r \rightarrow-\infty}\left|B_{r}^{0}\right|=\lim _{r \rightarrow-\infty}(\sqrt[4]{2})^{r}=0$. Moreover, for $n<0$

$$
\sum_{s=0}^{\infty}\left|B_{n-s+1}^{n} a_{n-s}\right|^{\alpha}=\frac{\sqrt[4]{2^{\alpha}}}{\sqrt[4]{2^{\alpha}}-1}
$$

and for $n \geq 0$ we obtain

$$
\begin{aligned}
\sum_{s=0}^{\infty}\left|B_{n-s+1}^{n} a_{n-s}\right|^{\alpha} & =\sum_{s=0}^{n}\left|B_{n-s+1}^{n} a_{n-s}\right|^{\alpha}+\sum_{s=n+1}^{\infty}\left|B_{n-s+1}^{n} a_{n-s}\right|^{\alpha} \\
& =\sum_{s=0}^{n}\left|a_{n-s}\right|^{\alpha}+\sum_{s=n+1}^{\infty}\left|B_{n-s+1}^{n}\right|^{\alpha} \\
& =\sum_{s=0}^{n} \frac{1}{2^{n-s}}+\sum_{s=n+1}^{\infty}\left(\frac{1}{\sqrt[4]{2}}\right)^{\alpha(s-n-1)} \\
& =\frac{1}{2^{n}} \sum_{s=0}^{n} 2^{s}+\sum_{s=0}^{\infty}\left(\frac{1}{\sqrt[4]{2}}\right)^{\alpha s}=2-\frac{1}{2^{n}}+\frac{\sqrt[4]{2^{\alpha}}}{\sqrt[4]{2^{\alpha}}-1} .
\end{aligned}
$$

From (3.9) and (3.10) we have $\sup _{n \in Z} \sum_{s=0}^{\infty}\left|B_{n-s+1}^{n} a_{n-s}\right|^{\alpha}<\infty$. So condition (I) is satisfied. We see at once that $B_{n+1}^{n+k}=1$ for $n \geq-1, k>0$, and $B_{n+1}^{n+k}=$ $(\sqrt[4]{2})^{n+1}$ for $n<-1, k \geq-n$. Therefore, for $1<\alpha \leq 2$ and $n \in Z$, one has:

$$
\begin{aligned}
& \lim _{k \rightarrow \infty} \frac{C D\left(X_{n}, X_{n+k}\right)}{C V\left(X_{n}, X_{n+k}\right)}= \begin{cases}2 & \text { for } n \geq-1, \\
\frac{1+(\sqrt[4]{2})^{-\alpha(n+1)}-\left|1-(\sqrt[4]{2})^{-n-1}\right|^{\alpha}}{(\sqrt[4]{2})^{-n-1}} & \text { for } n<-1,\end{cases} \\
& \lim _{k \rightarrow \infty} \frac{C D\left(X_{n+k}, X_{n}\right)}{C V\left(X_{n+k}, X_{n}\right)}= \begin{cases}2 & \text { for } n \geq-1, \\
\frac{1+(\sqrt[4]{2})^{\alpha(n+1)}-\left|1-(\sqrt[4]{2})^{n+1}\right|^{\alpha}}{(\sqrt[4]{2})^{n+1}} & \text { for } n<-1\end{cases}
\end{aligned}
$$

It is clear that these two limits depend on $n$.

(b) Let the coefficients differ slightly from those in (a):

$$
b_{n}=\left\{\begin{array}{ll}
-1 & \text { for } n \geq 0, \\
\frac{1}{\sqrt[4]{2}} & \text { for } n<0,
\end{array} \quad a_{n}= \begin{cases}\left(\frac{1}{2}\right)^{n / \alpha} & \text { for } n \geq 0 \\
1 & \text { for } n<0\end{cases}\right.
$$

It is obvious that condition (I) is satisfied and $B_{n+1}^{n+k}=(-1)^{k}$ for $n \geq$ $-1, k>0$, and $B_{n+1}^{n+k}=(-1)^{k}(-\sqrt[4]{2})^{n+1}$ for $n<-1, k \geq-n$. Hence for 
$1<\alpha \leq 2$ and $n \in Z$ one has:

$$
\begin{aligned}
& \frac{C D\left(X_{n}, X_{n+k}\right)}{C V\left(X_{n}, X_{n+k}\right)} \\
& = \begin{cases}2^{\alpha}-2 & \text { for } n \geq-1, k=1,3,5, \ldots, \\
2 & \text { for } n \geq-1, k=2,4,6, \ldots, \\
\frac{1+(\sqrt[4]{2})^{-\alpha(n+1)}-\left|1-(-1)^{k}(-\sqrt[4]{2})^{-n-1}\right|^{\alpha}}{(-1)^{k}(-\sqrt[4]{2})^{-n-1}} & \text { for } n<-1, k \geq-n,\end{cases} \\
& \frac{C D\left(X_{n+k}, X_{n}\right)}{C V\left(X_{n+k}, X_{n}\right)} \\
& \quad= \begin{cases}2^{\alpha}-2 & \text { for } n \geq-1, k=1,3,5, \ldots, \\
2 & \text { for } n \geq-1, k=2,4,6, \ldots, \\
\frac{1+(\sqrt[4]{2})^{\alpha(n+1)}-\left|1-(-1)^{k}(-\sqrt[4]{2})^{n+1}\right|^{\alpha}}{(-1)^{k}(-\sqrt[4]{2})^{n+1}} & \text { for } n<-1, k \geq-n .\end{cases}
\end{aligned}
$$

We see at once that $\operatorname{limits}_{k \rightarrow \infty} \frac{C D\left(X_{n}, X_{n+k}\right)}{C V\left(X_{n}, X_{n+k}\right)}$ and $\lim _{k \rightarrow \infty} \frac{C D\left(X_{n+k}, X_{n}\right)}{C V\left(X_{n+k}, X_{n}\right)}$ do not exist for considered coefficients.

\section{AR(1) MODELS SATISFYING CONDITION (II)}

If we assume that condition (II) is satisfied and $1<\alpha \leq 2$, then it can be easily shown that system (1.1) has the bounded solution in space $S_{\alpha}$ with norm $\|\cdot\|_{\alpha}$ given by formula:

$$
X_{n}=-\sum_{s=1}^{\infty} \frac{a_{n+s}}{B_{n+1}^{n+s}} \xi_{n+s}
$$

and $X_{n}$ converges a.s.

As in the case of condition (I), the covariation and the codifference will be studied and the asymptotic relation between these two measures of dependence for the considered model will be examined. In Proposition 4.1, which is a consequence of Proposition 3.5.2 in Samorodnitsky and $\mathrm{Taqqu}^{[12]}$, we give the formulae for the covariation and the codifference for AR(1) models with coefficients satisfying condition (II).

Proposition 4.1. If $\lim _{r \rightarrow \infty}\left|B_{0}^{r}\right|^{-1}=0$, $\sup _{n \in Z} \sum_{s=1}^{\infty}\left|\frac{a_{n+s}}{B_{n+1}^{n+s}}\right|^{\alpha}<\infty$ (condition (II) ) and $\left\{X_{n}\right\}$ is the bounded solution of (1.1), then for $1 \stackrel{n+1}{<} \alpha 2$ :

(i) the covariation of $X_{n}$ on $X_{m}(n, m \in Z)$ has the following form:

$$
C V\left(X_{n}, X_{m}\right)=\sigma_{\xi}^{\alpha}\left(B_{\min (n, m)+1}^{\max (n, m)}\right)^{\operatorname{sign}(n-m)} \sum_{s=-\infty}^{\min (-1, n-m-1)}\left|\frac{a_{n-s}}{B_{m+1}^{n-s}}\right|^{\alpha},
$$


(ii) the codifference of $X_{n}$ on $X_{m}(n, m \in Z)$ has the following form:

$$
\begin{aligned}
C D\left(X_{n}, X_{m}\right)= & \sigma_{\xi}^{\alpha}\left(1+\left|B_{\min (n, m)+1}^{\max (n, m)}\right|^{\alpha \cdot \operatorname{sign}(n-m)}-\left|1-\left(B_{\min (n, m)+1}^{\max (n, m)}\right)^{\operatorname{sign}(n-m)}\right|^{\alpha}\right) \\
& \times \sum_{s=-\infty}^{\min (-1, n-m-1)}\left|\frac{a_{n-s}}{B_{m+1}^{n-s}}\right|^{\alpha}
\end{aligned}
$$

Example 4.1 (The Case of Constant Coefficients). Let us consider the special case of $\mathrm{AR}(1)$ system, i.e., model with constant coefficients, given by the equation:

$$
X_{n}-b X_{n-1}=a \xi_{n}
$$

where $a \neq 0,|b|>1$ are real numbers and $\left\{\xi_{n}\right\}$ are independent $\mathrm{S} \alpha \mathrm{S}$ innovations with the scale parameter $\sigma_{\xi}$. As in Example 3.1, we can show that in this case condition (II) is satisfied and

$$
X_{n}=-a \sum_{s=1}^{\infty} \frac{\xi_{n+s}}{b^{s}}=-a \sum_{s=-\infty}^{-1} b^{s} \xi_{n-s}
$$

Moreover, it is not difficult to show that for $1<\alpha<2$ and for every $n$ we obtain the following limits:
(a) $\lim _{k \rightarrow \infty} \frac{C D\left(X_{n+k}, X_{n}\right)}{C V\left(X_{n+k}, X_{n}\right)}=0$
(c) $\lim _{k \rightarrow \infty} \frac{C D\left(X_{n-k}, X_{n}\right)}{C V\left(X_{n-k}, X_{n}\right)}=\alpha$,
(b) $\lim _{k \rightarrow \infty} \frac{C D\left(X_{n}, X_{n-k}\right)}{C V\left(X_{n}, X_{n-k}\right)}=0$
(d) $\lim _{k \rightarrow \infty} \frac{C D\left(X_{n}, X_{n+k}\right)}{C V\left(X_{n}, X_{n+k}\right)}=\alpha$.

And now again, the following question arises: Are the results (a)-(d) in Example 4.1 true in general in the case considered in this section? The answer is given in Theorem 5.1 and Example 5.2. Theorem 5.1 is presented without proof because of its similarity to the proof of Theorem 3.1.

Theorem 4.1. If $\lim _{r \rightarrow \infty}\left|B_{0}^{r}\right|^{-1}=0$, $\sup _{n \in Z} \sum_{s=1}^{\infty}\left|\frac{a_{n+s}}{B_{n+1}^{n+s}}\right|^{\alpha}<\infty \quad$ (condition (II)) and $\left\{X_{n}\right\}$ is a bounded solution of system (1.1), then:

(i) for $1<\alpha \leq 2$ and $n \in Z$ one has

$$
\lim _{k \rightarrow \infty} \frac{C D\left(X_{n}, X_{n+k}\right)}{C V\left(X_{n}, X_{n+k}\right)}=\alpha,
$$


(ii) for $1<\alpha<2$ and $n \in Z$ the following formula holds:

$$
\lim _{k \rightarrow \infty} \frac{C D\left(X_{n+k}, X_{n}\right)}{C V\left(X_{n+k}, X_{n}\right)}=0
$$

Example 4.2. Let us take into account the $\mathrm{AR}(1)$ system with timevarying coefficients given by (1.1) with $\mathrm{S} \alpha \mathrm{S}$ innovations (1.2) and consider two paricular cases of coefficients.

(a) The coefficients are given by

$$
b_{n}=\left\{\begin{array}{ll}
\sqrt[4]{2} & \text { for } n \geq 0, \\
1 & \text { for } n<0,
\end{array} \quad a_{n}= \begin{cases}1 & \text { for } n \geq 0 \\
2^{n / \alpha} & \text { for } n<0\end{cases}\right.
$$

An easy computation shows that these coefficients satisfy condition (II). Moreover, it is a simple matter to notice that $B_{n-k+1}^{n}=1$ for $n \leq-1, k>0$, and $B_{n-k+1}^{n}=(\sqrt[4]{2})^{n+1}$ for $n>-1, k \geq n+1$ and hence, by Proposition 4.1, for $1<\alpha \leq 2$ and $n \in Z$ one has:

$$
\begin{aligned}
& \lim _{k \rightarrow \infty} \frac{C D\left(X_{n}, X_{n-k}\right)}{C V\left(X_{n}, X_{n-k}\right)}= \begin{cases}2 & \text { for } n \leq-1, \\
\frac{1+(\sqrt[4]{2})^{\alpha(n+1)}-\left|1-(\sqrt[4]{2})^{n+1}\right|^{\alpha}}{(\sqrt[4]{2})^{n+1}} & \text { for } n>-1,\end{cases} \\
& \lim _{k \rightarrow \infty} \frac{C D\left(X_{n-k}, X_{n}\right)}{C V\left(X_{n-k}, X_{n}\right)}= \begin{cases}2 & \text { for } n \leq-1, \\
\frac{1+(\sqrt[4]{2})^{-\alpha(n+1)}-\left|1-(\sqrt[4]{2})^{-n-1}\right|^{\alpha}}{(\sqrt[4]{2})^{-n-1}} & \text { for } n>-1 .\end{cases}
\end{aligned}
$$

It is obvious that these two limits are dependent on $n$ and for $\operatorname{AR}(1)$ models with time-varying coefficients we do not have the same results like in the stationary case obtained in Example 4.1.

(b) Let the coefficients differ slightly from those in (a):

$$
b_{n}=\left\{\begin{array}{ll}
\sqrt[4]{2} & \text { for } n \geq 0, \\
-1 & \text { for } n<0,
\end{array} \quad a_{n}= \begin{cases}1 & \text { for } n \geq 0 \\
2^{n / \alpha} & \text { for } n<0\end{cases}\right.
$$

It is not difficult to show that condition (II) is satisfied and that for $1<\alpha \leq 2$ and $n \in Z$ we obtain the following quotients:

$$
\begin{aligned}
& \frac{C D\left(X_{n}, X_{n-k}\right)}{C V\left(X_{n}, X_{n-k}\right)} \\
& \quad= \begin{cases}2^{\alpha}-2 & \text { for } n \leq-1, k=1,3,5, \ldots, \\
2 & \text { for } n \leq-1, k=2,4,6, \ldots, \\
\frac{1+(\sqrt[4]{2})^{\alpha(n+1)}-\left|1-(-1)^{k}(-\sqrt[4]{2})^{n+1}\right|^{\alpha}}{(-1)^{k}(-\sqrt[4]{2})^{n+1}} & \text { for } n>-1,\end{cases}
\end{aligned}
$$


TABLE 1 Asymptotic relationship between covariation and codifference for both considered conditions

\begin{tabular}{llr}
\hline Limit & (I) & (II) \\
\hline $\lim _{k \rightarrow \infty} \frac{C D\left(X_{n}, X_{n-k}\right)}{C V\left(X_{n}, X_{n-k}\right)}$ & $\alpha$ & $\star$ \\
$\lim _{k \rightarrow \infty} \frac{C D\left(X_{n-k}, X_{n}\right)}{C V\left(X_{n-k}, X_{n}\right)}$ & 0 & $\star$ \\
$\lim _{k \rightarrow \infty} \frac{C D\left(X_{n}, X_{n+k}\right)}{C V\left(X_{n}, X_{n+k}\right)}$ & $\star$ & \\
$\lim _{k \rightarrow \infty} \frac{C D\left(X_{n+k}, X_{n}\right)}{C V\left(X_{n+k}, X_{n}\right)}$ & $\star$ & \\
\hline “”- the limit exists (but in some cases it depends \\
on $n)$ or does not exist, see Examples 3.2 and 4.2.
\end{tabular}

$$
\begin{aligned}
& \frac{C D\left(X_{n-k}, X_{n}\right)}{C V\left(X_{n-k}, X_{n}\right)} \\
& \quad= \begin{cases}2^{\alpha}-2 & \text { for } n \leq-1, k=1,3,5, \ldots, \\
2 & \text { for } n \leq-1, k=2,4,6, \ldots, \\
\frac{1+(\sqrt[4]{2})^{-\alpha(n+1)}-\left|1-(-1)^{k}(-\sqrt[4]{2})^{-n-1}\right|^{\alpha}}{(-1)^{k}(-\sqrt[4]{2})^{-n-1}} & \text { for } n>-1 .\end{cases}
\end{aligned}
$$

Therefore, it is obvious that for considered coefficients both limits $\lim _{k \rightarrow \infty} \frac{C D\left(X_{n}, X_{n-k}\right)}{C V\left(X_{n}, X_{n-k}\right)}$ and $\lim _{k \rightarrow \infty} \frac{C D\left(X_{n-k}, X_{n}\right)}{C V\left(X_{n-k}, X_{n}\right)}$ do not exist.

\section{CONCLUSION}

In this paper $\mathrm{AR}(1)$ systems with $\mathrm{S} \alpha \mathrm{S}$ innovations and time-dependent coefficients have been considered. Conditions providing the bounded solutions of such systems have been formulated and the form of the solution has been stated (these results generalize the results of Makagon et al. $\left.^{[5]}\right)$. Then the formulae obtained in Nowicka ${ }^{[8]}$ and Nowicka and Weron $^{[9]}$ in case of stationary ARMA models with $\mathrm{S} \alpha \mathrm{S}$ innovations has been extended to non-stationary case of $\mathrm{AR}(1)$ system (Theorems 3.1 and 4.1). Moreover, some examples have been presented to show that in the case of time-varying coefficients the asymptotic relation between the codifference and the covariation differs from outcomes obtained in the case of constant ones. The main results concerning the asymptotic relation between considered measures of dependence are collected in Table 1.

The study carried out in this paper open up a new areas of interest for non-stationary models with the innovations different than Gaussian ones. 


\section{REFERENCES}

1. Gardner, W.; Franks, L.E. Characterization of cyclostationary random signal processes. IEEE Transactions of Information Theory 1975, 21, 4-14.

2. Gladyshev, E.G. Periodically correlated random sequences. Sov. Math. 1961, 2, 385-388.

3. Janicki, A.; Weron, A. Can one see $\alpha$-stable variables and processes? Statist. Sci. 1994, 9, 109-126.

4. Jones, R.H.; Brelsford, W.M. Time series with periodic structure. Biometrika 1967, 54, 403-408.

5. Makagon, A.; Weron, A.; Wyłomańska, A. Bounded solutions for ARMA model with varying coefficients. Appl. Math. 2004, 31, 273-285.

6. Mittnik, S.; Rachev, S.T. Stable Paretian Models in Finance; Wiley: New York, 2000.

7. Monin, A.S. Stationary and periodic time series in the general circulation of the atmosphere. In Proceedings Symposium on Time Series Analysis; Rosenblatt, M., Ed.; 1963; 144-151.

8. Nowicka, J. Asymptotic behavior of the covariation and the codifference for ARMA models with stable innovations. Stochastic Models 1997, 13, 673-685.

9. Nowicka, J.; Weron, A. Measures of dependence for ARMA Models with stable innovations, Annales UMCS Section A 1997, LI, 1, 14, 133-144.

10. Nowicka-Zagrajek, J.; Wyłomańska, A. The dependence structure for PARMA models with $\alpha$-stable innovations. Acta Physica Polonica B 2006, 37 (11), 3071-3082.

11. Parzen, E.; Pagano, M. An approach to modeling seasonally stationary time series. Journal of Econometrics 1979, 9, 137-153.

12. Samorodnitsky, G.; Taqqu, M.S. Stable Non-Gaussian Random Processes; Chapman \& Hall: New York, 1994.

13. Stuck, B.W.; Kleiner, B. A statistical analysis of telephone noise. Bell System Technical Journal 1974, 53, 1263-1320.

14. Vecchia, A.V. Periodic autoregressive-moving average (PARMA) modeling with applications to water resources. Water Resources Bulletin 1985, 21, 721-730.

15. Vecchia, A.V. Maximum likelihood estimation for periodic autoregressive-moving average models. Technometrics 1985, 27, 375-384. 